\title{
Evaporation-Induced Diffusion Acceleration In Liquid-Filled Porous Materials
}

Xuefeng Wang ${ }^{a, b}$, Pengpeng Jia ${ }^{a, b}$, Shanyouming Sun ${ }^{b}$, Xiaocong He ${ }^{a, b}$, Tian Jian Lu ${ }^{c, d}$, Feng $X u^{a, b}$, Shangsheng Feng ${ }^{a, b^{*}}$

${ }^{a}$ The Key Laboratory of Biomedical Information Engineering of Ministry of Education, School of Life Science and Technology, Xi'an Jiaotong University, Xi'an 710049, P.R. China

${ }^{b}$ Bioinspired Engineering and Biomechanics Center (BEBC), Xi'an Jiaotong University, Xi'an 710049, P.R. China

${ }^{c}$ State Key Laboratory of Mechanics and Control of Mechanical Structures, Nanjing University of Aeronautics and Astronautics, Nanjing 210016, P.R. China

${ }^{d}$ Nanjing Center for Multifunctional Lightweight Materials and Structures (MLMS), Nanjing University of Aeronautics and Astronautics, Nanjing 210016, P.R. China

*Corresponding author: shangshengfeng@xjtu.edu.cn 


\section{Table of contents}

Section 1: Experimental Results for Pure Diffusion Without Evaporation

Section 2: Simulation of Evaporation Flux 


\section{Supporting Information}

\section{Section 1: Experimental Results for Pure Diffusion Without Evaporation}

Using dual environmental chambers, we achieved approximately $100 \%$ relative humidity around the NC membrane to minimize evaporation effects. In this case, diffusion was driven only by concentration gradients of erioglaucine disodium salt dye along the NC membrane. Firstly, two NC membranes (HFC13502, HFC07502), with different specifications (e.g., porosity, pore size), were tested to investigate their diffusion behaviors without evaporation. The measured concentration distributions of erioglaucine disodium salt dye are presented in Figure S1 together with theoretical model predictions, at the instant time of 20 minutes after diffusion was initialed. The concentration profiles decrease sharply with increasing diffusion distance, and the experimental data are consistent with the classic 1-D diffusion theory without evaporation. The good agreement validates our experimental method.

For both NC membranes, the insert of Figure S1 presents the effective diffusion coefficient $D_{\text {eff }}$ fitted at different diffusion times. It was found that, approximately speaking, $D_{\text {eff }}$ does not change with time, indicating negligible interception or adsorption of dye on NC membrane skeleton, for the average pore diameter $(4-9 \mu \mathrm{m})$ is three orders of magnitude higher than the diameter of dye $(\sim 10 \mathrm{~nm})$. To verify whether dye molecules were adsorbed by NC membranes framework, we carried out separate experiments, by first immersing the NC membrane for $\sim 3$ hours in the erioglaucine disodium salt dye solution, and then placing it in deionized (DI) water for $\sim 3$ hours. ${ }^{1}$ We found that the two NC membranes were completely faded and the dye molecules were completely diffused, indicating that there was no adsorption between erioglaucine disodium salt dye molecules and NC membranes. In addition, the measured effective diffusion coefficient is $10^{-10} \mathrm{~m}^{2} / \mathrm{s}$, the same order as the reported values of hydrogel and $\mathrm{NC}$ membranes in open literature, ${ }^{2-4}$ which further demonstrates the credibility of our experimental results. 


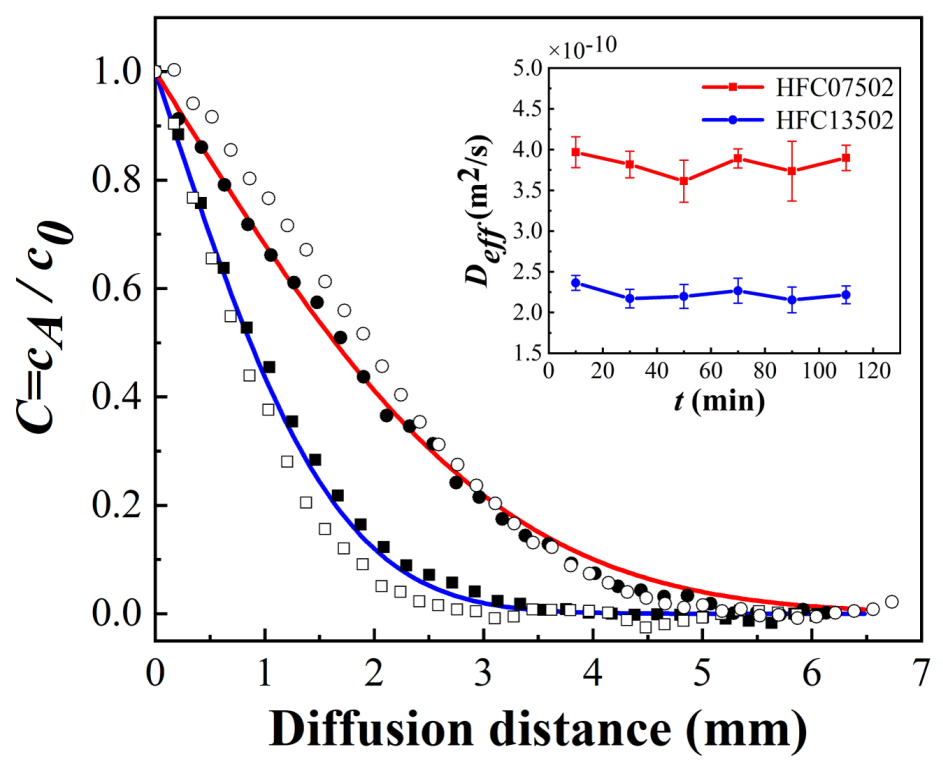

Figure S1. Concentration distributions of diffusing substance in two liquid-filled NC membranes in the absence of evaporation, the solid and the blank symbols represent two parallel experiments. Illustration: effective diffusion coefficients of two NC membranes fitted at different times. 


\section{Section 2: Simulation of Evaporation Flux}

Evaporation flux on the surface of a NC membrane was obtained by solving the vapor diffusion equation. Given that the saturated vapor pressure of water is much higher than that of erioglaucine disodium salt dye solute, according to Raoult's law, the diffusion substance is only water vapor, and vapor diffusion is caused by gradient of vapor concentration between the surface of the NC membrane and the environment.

Based on the above assumption, a three-dimensional (3-D) diffusion equation was adopted for modeling vapor concentration distribution in environmental chamber, as:

$\frac{\partial c_{\mathrm{w}}}{t}+\nabla \cdot J_{\mathrm{w}}=0$

where $c_{\mathrm{w}}$ is the vapor concentration and $J_{\mathrm{w}}$ is the diffusion flux of vapor in the chamber.

The computational domain was the environmental chamber with the same size as that used in experiment, as shown in Figure S2a. The bottom of the environment chamber was set as no flux boundary condition; environmental constant vapor concentration $c_{\infty}=\mathrm{RH} c_{\mathrm{s}}$ was applied to both the side and top walls, where $c_{\mathrm{s}}$ was the saturated vapor concentration at $21^{\circ} \mathrm{C}$, which was $2.03 \times 10^{-2} \mathrm{~kg} / \mathrm{m}^{3}$. ${ }^{5}$ Upon ignoring the tortuosity of NC membrane surface, the corresponding boundary conditions on the surface was set as $c_{\mathrm{s}}$. The initial vapor concentration in the chamber was set as $\mathcal{c}_{0 .}{ }^{6-9}$

(a)

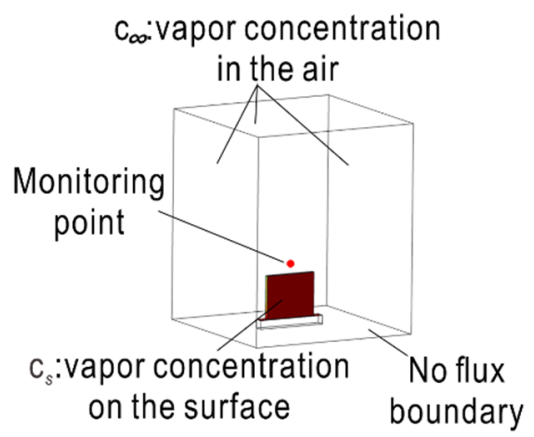

(b)

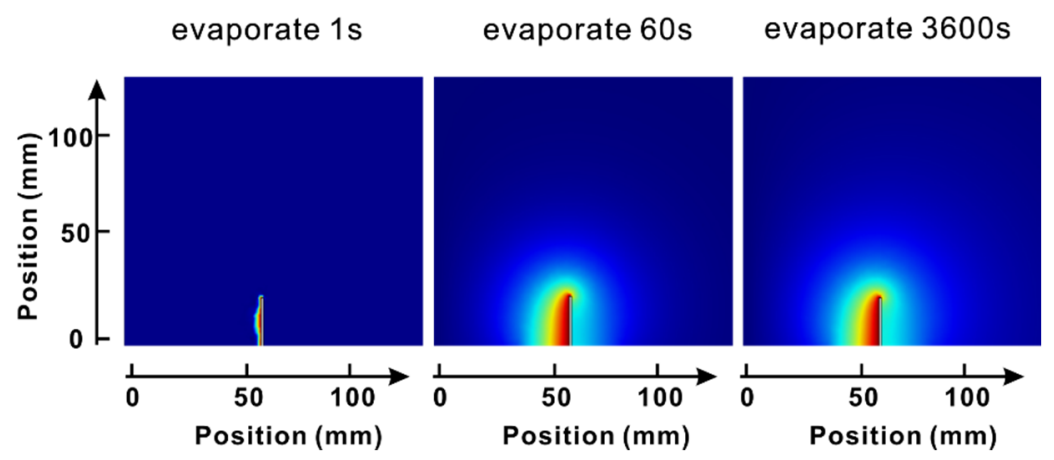

Figure S2. Simulation of evaporation flux on the NC membranes surface: (a) computational domain and boundary conditions; (b) simulated vapor concentration distributions.

Once eq 1 was solved with the above boundary conditions, average evaporation flux from the surface of NC membrane was obtainable. The simulation result was plotted as a function of 
diffusion time, as shown in Figure S3a. The simulated average evaporation flux was fitted according to second-order exponential function, as:

$m_{\mathrm{e}}=a \cdot e^{(b t)}+c \cdot e^{(d t)}$

which was then used to determine flow velocity at the root of $\mathrm{NC}$ membrane. The fitting results are also shown in Figure S3a.

To validate the 3-D evaporation diffusion model, a temperature and humidity prober (Figure S2a) was located nearby the test sample to monitor the transient responses of temperature and $\mathrm{RH}$ in the chamber. The measured RH history shown in Figure S3b agreed well with simulated results, thus validating the present numerical model.

(a)

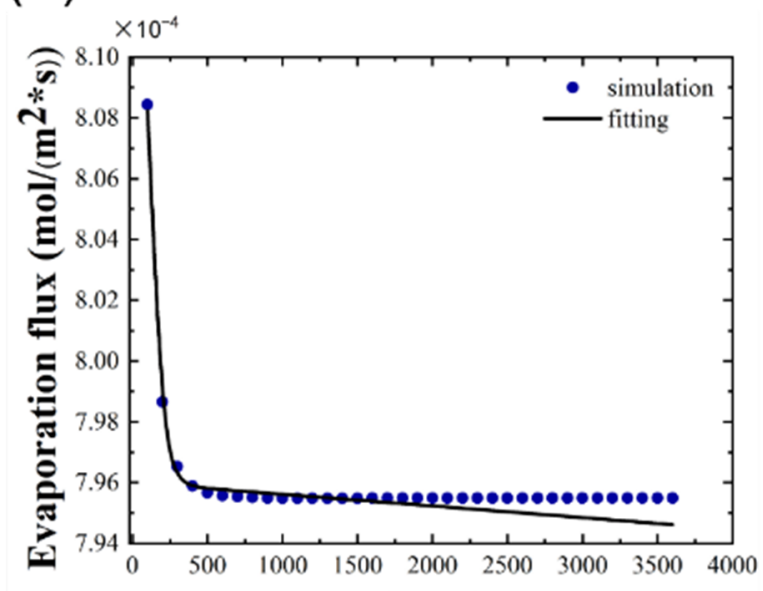

Time (s) (b)

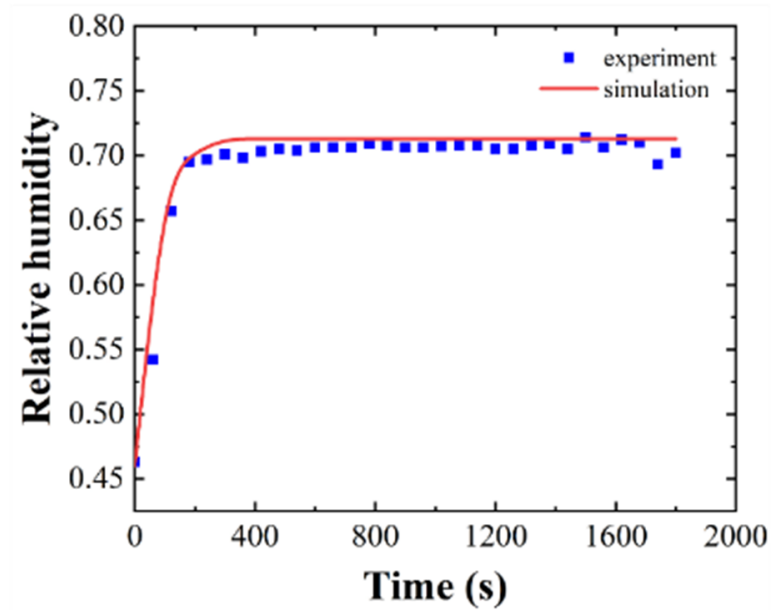

Figure S3. (a) Simulated average evaporation flux $m_{\mathrm{e}}$ plotted as a function of time $t$ and (b) comparison of RH at selected point between experimental and simulated results. 


\section{REFERENCES}

1. Liu, D.; Kotsmar, C.; Nguyen, F.; Sells, T.; Taylor, N.; Prausnitz, J.; Radke, C., Macromolecule sorption and diffusion in HEMA/MAA hydrogels. Ind. Eng. Chem. Res. 2013, 52 (50), 18109-18120.

2. Samprovalaki, K.; Fryer, P. J., Development of an experimental procedure for real time investigation of diffusion in foods. Sensing Instrumentation for Food Quality Safety 2011, 5 (2), 78-89.

3. Ahmad, A.; Low, S.; Shukor, S. A.; Fernando, W.; Ismail, A. J. J. o. M. S., Hindered diffusion in lateral flow nitrocellulose membrane: Experimental and modeling studies. $J$. Membr. Sci. 2010, 357 (1-2), 178-184.

4. Perullini, M.; Jobbágy, M.; Japas, M. L.; Bilmes, S. A., New method for the simultaneous determination of diffusion and adsorption of dyes in silica hydrogels. J. Colloid Interface Sci. 2014, 425, 91-95.

5. Haynes, W. M., CRC handbook of chemistry and physics. CRC press: 2014.

6. Pradhan, T. K.; Panigrahi, P. K., Unexpectedly absence of Marangoni convection in an evaporating water meniscus. arXiv preprint arXiv:2004.01730 2020.

7. Pradhan, T. K.; Panigrahi, P. K., Condensation induced internal convection of two neighboring droplets. arXiv preprint arXiv:2003.09013 2020.

8. Li, Y.; Lv, P.; Diddens, C.; Tan, H.; Wijshoff, H.; Versluis, M.; Lohse, D., Evaporationtriggered segregation of sessile binary droplets. Phys. Rev. Lett. 2018, 120 (22).

9. Diddens, C.; Tan, H.; Lv, P.; Versluis, M.; Kuerten, J. G. M.; Zhang, X.; Lohse, D., Evaporating pure, binary and ternary droplets: thermal effects and axial symmetry breaking. $J$. Fluid Mech. 2017, 823, 470-497. 